\title{
Observational Constraints on Trojans of Transiting Extrasolar Planets
}

\author{
Eric B. Ford ${ }^{1,2}$ and B. Scott Gaudi ${ }^{2,3}$ \\ eford, sgaudi@cfa.harvard.edu
}

\begin{abstract}
Theoretical studies predict that Trojans are likely a frequent byproduct of planet formation and evolution. We present a novel method of detecting Trojan companions to transiting extrasolar planets which involves comparing the midtime of eclipse with the time of the stellar reflex velocity null. We demonstrate that this method offers the potential to detect terrestrial-mass Trojans using existing ground-based observatories. This method rules out Trojan companions to HD 209458b and HD 149026b more massive than $\simeq 13 M_{\oplus}$ and $\simeq 25 M_{\oplus}$ at a $99.9 \%$ confidence level. Such a Trojan would be dynamically stable, would not yet have been detected by photometric or spectroscopic monitoring, and would be unrecognizable from radial velocity observations alone. We outline the future prospects for this method, and show that the detection of a "Hot Trojan" of any mass would place a significant constraint on theories of orbital migration.
\end{abstract}

Subject headings: techniques: photometric, radial velocities — planetary systems: formation - celestial mechanics

\section{Introduction}

Stable Trojan companions to extrasolar planets may be common. In our solar system, Mars, Jupiter, and Neptune share their orbit with asteroids orbiting near the stable (L4/L5) Lagrange points that lead/trail the planet by $\simeq 60^{\circ}$. Orbits near the L4/L5 points of the terrestrial planets, Saturn and Uranus are less stable due to perturbations from the other planets (Nesvorny \& Dones 2002). Saturn's satellites also include small moons orbiting about the L4/L5 points of Tethys and Dione. While the mass ratios of the Trojan systems in our solar system are rather extreme $\left(\leq 7 \times 10^{-9}\right)$, extrasolar planets may have more massive Trojans. Theorists have already outlined several mechanisms to form Trojans with mass ratios as large as unity. For example, Laughlin \& Chambers (2002) present hydrodynamic simulations of a protoplanetary disk where disk material lingers near the L4 and L5 points of a planet (near the gap-opening threshold). The resulting

\footnotetext{
${ }^{1}$ Hubble Fellow

${ }^{2}$ CfA, MS 51, 60 Garden St., Cambridge, MA 02138

${ }^{3}$ Current address: Department of Astronomy, The Ohio State University, 140 W. 18th Ave., Columbus, OH 43210
} 
vortex could trap particles and lead to the accretion of a Trojan in situ (Chiang \& Lithwick 2005). If disk torques caused the planet to gradually migrate inwards, then the Trojan would migrate with the planet. Unlike resonant migration in the 2:1 mean motion resonance, the eccentricity or libration amplitude of the Trojan would not be excited by the migration (Laughlin \& Chambers 2002). Alternatively, a body could be captured into an orbit about the L4/L5 point after a violent event, as has been suggested for the formation of Jupiter and Neptune Trojans in our solar system (Morbidelli et al. 2005). Capture into a Trojan orbit could also occur due to rapid mass growth of the planet or a collision of two objects near L4/L5 (Chiang \& Lithwick 2005 and reference therein). Another possibility is that convergent migration could trap multiple protoplanets into a 1:1 mean motion resonance (Thommes 2005, Cresswell \& Nelson 2006). In each of these scenarios, the captured bodies could initially have a large libration amplitude or reside on horseshoe-type orbits. However, if the capture occurred before or during the planet's inward migration, then interactions with either a gaseous or planetesimal disk would damp the libration amplitude. This mechanism is even capable of causing objects initially on horseshoe orbits to evolve into tadpole orbits and then small amplitude libration near the L4/L5 fixed point. Such behavior has been found in numerical simulations of multiple planet systems interacting with either a gaseous or planetesimal disk (Cresswell \& Nelson 2006; Ford \& Chiang 2006).

Trojans of Jupiter and Neptune have provided clues about our solar system's history (Michtchenko, Beauge \& Roig 2001; Kortenkamp, Malhotra \& Michtchenko 2004; Chiang \& Lithwick 2005; Morbidelli et al. 2005). Similarly, the detection of extrasolar Trojans would be useful for constraining theories of planet formation. While all the above mechanisms predict that Trojans would survive the migration process, there are alternative models of planet migration that predict Trojans would not survive. For example, while planet formation models generally agree that planets should form on nearly circular orbits, it is possible that gravitational perturbations by other planets or a binary companion could excite sizable eccentricities. One possible formation mechanism for short-period giant planets is that a planet acquires a large eccentricity (e.g., due to strong planet-planet scattering, secular perturbations from a binary companion, or being tidal captured) and comes so close to the star that tidal dissipation circularizes the orbit at a small semimajor axis (Rasio \& Ford 1996, Wu \& Murray 2003, Gaudi 2003, Ford \& Rasio 2006). Detecting a Trojan companion to a short-period planet would present a serious challenge for these mechanisms for forming "hot Jupiters" and would imply that the planet in such a system was formed via migration through a dissipative disk. Thus, searching for extrasolar Trojans can test models of planet formation. Here, we present a method for detecting Trojan companions to extrasolar planets by combining RV and photometric observations of transiting extrasolar planets. We refer to all bodies librating about the L4/L5 fixed point of a planet as "Trojans" and focus our attention on Trojans that are significantly less massive than the currently known planet and not currently recognizable from radial velocity (RV) observations alone. 


\section{Observational Constraints on Trojans}

We denote the stellar mass $\left(m_{\star}\right)$, the planet mass $\left(m_{p}\right)$, and the Trojan mass $\left(m_{T}\right)$. Since the known transiting planets have short orbital periods and are subject to rapid eccentricity damping (Rasio et al. 1996), we initially assume the planet to be on a circular orbit about a star. Then a Trojan would orbit at one of the two fixed points, L4/L5, which lie along the orbit of the planet and lead/trail the planet by $60^{\circ}$. If there are no other bodies in the system, then the L4/L5 fixed points are stable if the ratio, $\mu=\left(m_{p}+m_{T}\right) /\left(m_{\star}+m_{p}+m_{T}\right)$, is less than a critical threshold $\mu_{c}$, where $0.03812 \leq \mu_{c} \leq 0.03852$ and $\mu_{c}$ depends on $\epsilon \equiv m_{T} /\left(m_{p}+m_{T}\right)$ (Murray \& Dermott 2000). If the Trojan resides exactly at the L4/L5 fixed point, then the direction of the vector sum of the forces exerted on the star by the planet and Trojan will lead/trail the force exerted on the star by the planet alone by an angle, $\phi$, such that $\tan \phi \simeq \sqrt{3} \epsilon /(2-\epsilon) \times(1+O(\mu))$ (Fig. 1).

More generally, for a Trojan that is librating about the L4/L5 fixed point, $\phi$ will vary by an angle $|\Delta \phi| \sim \Delta \phi_{\text {fast }}+\Delta \phi_{\text {slow }}$, where $\Delta \phi_{\text {fast }}$ varies on the orbital period of the planet $P$, and $\Delta \phi_{\text {slow }}$ varies on the secular timescale, $P_{l i b} \simeq P \sqrt{4 / 27} \mu^{-1 / 2}$ (Murray \& Dermott 2000). Since the Trojans of short period planets are likely to have formed in the presence of a dissipative disk, we focus on Trojans undergoing small librations with an amplitude, $\delta a \ll \mu^{1 / 2} a_{p}$, where $a_{p}$ is the semimajor axis of the planet. In this case, $\Delta \phi_{\text {fast }} \sim e_{T} \epsilon$, where $e_{T}$ is the Trojan's osculating eccentricity, and $\Delta \phi_{\text {slow }} \sim\left[\delta a /\left(6 \mu^{1 / 2} a\right)\right] \epsilon$. Nesvorny et al. (2002) show that the behavior of Trojans of planets with small eccentricities is similar. Perturbations (e.g., GR, stellar quadrupole, tides) are unlikely to affect the stability, since they typically have timescales much longer than the orbital or libration timescale.

If a planet on a circular orbit were the only body perturbing the central star, then the time that the stellar RV equals the RV of the system barycenter $\left(T_{0}\right)$ would coincide with the time of midtransit $\left(T_{c}\right)$. However, the gravitational perturbation of a Trojan at L4/L5 would cause these two times to differ by

$$
\begin{aligned}
\Delta t & \equiv T_{0}-T_{c}= \pm \frac{\phi P}{2 \pi} \simeq \pm \frac{\sqrt{3} \epsilon P}{4 \pi} \\
& \simeq \pm 37.5\left(\frac{P}{3 \mathrm{~d}}\right)\left(\frac{m_{T}}{10 m_{\oplus}}\right)\left(\frac{0.5 M_{J}}{m_{p}+m_{T}}\right) \mathrm{min} .
\end{aligned}
$$

A Trojan can signal its presence by a time offset between the ephemeris determined from transit photometry and the ephemeris determined from RVs (Fig. 1). If there are Trojans at both L4 and L5, then this measures the difference in mass at L4 and the mass at L5. If a planet is on a slightly eccentric orbit, then there is an offset of $\Delta t \simeq P /(2 \pi) \times\left(e \cos \omega+O\left(e^{2}\right)\right)$, where $\omega$ is the argument of pericenter, even in the absence of a Trojan. While short-period planets are expected to circularize rapidly, it is desirable to constrain the eccentricity observationally (e.g., by RV observations or timing of the secondary eclipse) before claiming the detection of a Trojan. Additional planets could also perturb the time of midtransit (Holman \& Murray 2005; Agol et al. 2005) so the offset will vary from transit to transit. Therefore multiple transits should be observed 
to verify that any observed offsets are not due to perturbations by a more distant planet.

For a transiting planet, both $P$ and $T_{c}$ can be measured precisely using photometry alone. Consider a series of continuous photometric observations with uncorrelated Gaussian uncertainties of magnitude $\sigma_{p h}$, taken at a rate $\Gamma$ around a single transit. The transit time can be measured with an accuracy of $\sigma_{T_{c}} \simeq \sqrt{t_{e} / 2 \Gamma} \sigma_{p h} \rho^{-2}$, where $t_{e}$ is the duration of ingress/egress and $\rho$ is the ratio of the planet radius to stellar radius. For typical parameters (e.g., $\sigma_{p h} \sim 10^{-3}$ ), $T_{c}$ can be measured to $\simeq 10$ s (e.g., Brown et al. 2001). The period can be measured much more accurately, from observations of multiple transits separated by many orbits.

Given the measurement precision for $T_{c}$ and $P$, the practical limit on measuring $\Delta t$ is set by the uncertainty in $T_{0}$ from RV observations. Assuming a circular orbit, the RV observations at a time $t_{i}$ can be fit by the mode $11 v_{i}=C+K \sin \left(2 \pi\left(t_{i}-T_{c}\right) / P+\phi\right)=C+A \sin \left(2 \pi\left(t_{i}-T_{c}\right) / P\right)+$ $B \cos \left(2 \pi\left(t_{i}-T_{c}\right) / P\right)$. where $A \equiv K \cos \phi, B \equiv K \sin \phi$, and $K$ is the velocity semi-amplitude. Assuming the period determined from photometric observations, the coefficients $A, B$, and $C$, (and hence the phase difference, $\tan \phi=B / A$ ) can be determined by linear least squares fitting to RV observations. If there are $N_{R V} \mathrm{RV}$ observations with uncorrelated Gaussian uncertainties $\left(\sigma_{R V}\right)$ and many RV observations are evenly distributed over orbital phase, then a Fisher information analysis (Gaudi \& Winn 2006) reveals that the uncertainties in model parameters will approach $\sigma_{A}=\sigma_{B}=\sqrt{2 / N} \sigma_{R V}, \sigma_{\phi}=\sqrt{2 / N_{R V}} \sigma_{R V} / K$, and $\sigma_{\Delta t} \simeq \sigma_{T_{0}}=\sqrt{1 / 2 \pi^{2} N_{R V}} P \sigma_{R V} / K$. A similar analysis for an eccentric orbit in the epicyclic approximation, shows that the uncertainty in $\Delta t$ is increased by a modest factor over the expression above. If a Trojan were present, then the uncertainty in $\phi$ would set the uncertainty in the measurement of the mass of the Trojan,

$$
\begin{aligned}
\sigma_{m_{T}} & =\frac{4 \pi}{\sqrt{3}} m_{P} \frac{\sigma_{\Delta t}}{P}=\sqrt{\frac{8}{3 N_{R V}}} m_{P} \frac{\sigma_{R V}}{K} \\
& =0.52 M_{\oplus}\left(\frac{50}{N_{R V}}\right)^{1 / 2}\left(\frac{\sigma_{R V}}{\mathrm{~m} \mathrm{~s}^{-1}}\right)\left(\frac{P}{3 \mathrm{~d}}\right)^{1 / 3}\left(\frac{m_{\star}}{M_{\odot}}\right)^{2 / 3} .
\end{aligned}
$$

If we were to demand a measurement of $\Delta t>3.291 \sigma_{\Delta t}$ to claim the detection of a Trojan, then a total of $\simeq 160(60)$ precision $\mathrm{RV}$ measurements could detect $\mathrm{a} \simeq 3 M_{\oplus}\left(5 M_{\oplus}\right)$ Trojan, assuming a host star with an intrinsic jitter, $\sigma_{j} \simeq 3 \mathrm{~m} \mathrm{~s}^{-1}$ (Wright 2005), and $1 \mathrm{~m} \mathrm{~s}^{-1}$ measurement uncertainties added in quadrature. While challenging, it is remarkable that current ground based instruments have the necessary precision to detect such a low mass Trojan with a plausible amount of observing time.

\footnotetext{
${ }^{1}$ Ignoring any observations during primary transit when the Rossiter-McLaughlin effect distorts the observed RV (Winn et al. 2005; Gaudi \& Winn 2006).
} 


\section{Example Application}

In Table 1, we summarize the current observational parameters and sensitivity to Trojan companions of extrasolar planets that transit bright stars, based on the above analysis. We find that combining the above method with existing observations already provides significant upper limits on the mass of Trojan companions to the planets HD 209458b and HD 149026b. Next, we perform more careful Bayesian analyses of the current observational constraints for these two cases. For HD 209458b we adopt the transit period and ephemeris of Knutson et al. (2006). We reanalyzed the RV measurements from Butler et al. (2006), fixing the orbital period and transit ephemeris. We use Markov chain Monte Carlo (Ford 2005, 2006; Gregory 2005) to sample from the posterior probability distribution for the remaining RV model parameters $K, e, \omega, M_{0}, C$, and $\sigma_{j}$, where $M_{0}$ is the mean anomaly at the epoch of midtransit. We assume priors that are flat in $\log \left(1+K / K_{o}\right), e, \omega, M_{0}, C$, and $\log \left(1+\sigma_{j} / \sigma_{o}\right)$, and choose $K_{o}=\sigma_{o}=1 \mathrm{~m} \mathrm{~s}^{-1}$, but our results are insensitive to these assumptions. We then construct the posterior distribution for the quantity $\left(M_{0}-e \cos \omega\right) P /(2 \pi) \simeq \Delta t$. In Fig. 2a we show the distributions for $\Delta t$ using three different assumptions. We find $\Delta t=-11.4 \pm 8.7 \mathrm{~min}$ (circular orbit), $\Delta t=-16.4 \pm 10.8 \mathrm{~min}$ (eccentric orbit ignoring the secondary eclipse), and $\Delta t=-13.1 \pm 8.9 \mathrm{~min}$ (eccentric orbit using the time of the secondary eclipse; Deming et al. 2005). We conclude that existing observations place an upper limit on the mass of Trojan companions to HD 209458b of $13.2 M_{\oplus}$ at the $99.9 \%$ confidence level.

We have performed a similar analysis of HD 149026b (Fig. 2b) using the observations of Butler et al. (2006) and Charbonneau et al. (2006). If we assume a circular (eccentric) orbit, then we find $\Delta t=-19 \pm 31 \mathrm{~min}(\Delta t=98 \pm 112 \mathrm{~min})$. The constraint is significantly weaker when we allow for an eccentric orbit, due to the limited number of RV observations and poor phase coverage. Incorporating a preliminary estimate of the time of the secondary eclipse (J. Harrington 2006, private communication), we find $\Delta t=13 \pm 27 \mathrm{~min}$ and place an upper limit on the mass of Trojan companions to HD $149026 \mathrm{~b}$ of $24.5 M_{\oplus}$ at the $99.9 \%$ confidence level.

\section{Discussion}

In principle, Trojans could be detected via their radial velocity, astrometric, transit, or transit timing signatures. If a Trojan is sufficiently massive and has a sufficiently large libration amplitude, then it could be detected from the deviations from a Keplerian perturbation to the stellar radial velocity or astrometric signal caused by a single planet. Laughlin \& Chambers (2002) have shown that two comparable mass planets occupying a 1:1 mean motion resonance would typically have strong planet-planet gravitational interactions on a secular timescale. However, these signatures may not be unique: a reanalysis of the RV observations of HD 128311 and HD 82943 have shown that both of the current data sets are consistent with a pair of planets in a 1:1 mean motion

resonance (Gozdziewski \& Konacki 2006), as well as the originally published orbital solutions involving higher-order mean motion resonances. 
Trojans may also be detectable if they transit their parent star. Photometric or spectroscopic monitoring of stars with transiting planets (particularly at times offset from the planet transit by $\sim P / 6$ ) may reveal the Trojan transit via the decrease in stellar flux or anomalous RV excursions due to the Rossiter-McLaughlin effect (Gaudi \& Winn 2006). For planets discovered via a photometric transit search, there will typically be observations at epochs useful for searching for large Trojans. Unfortunately, a Trojan might not transit its parent star if it has a significant inclination (e.g., Morbidelli et al. 2005). Since the libration period can be large, long-term monitoring would be required to ensure detection. Currently, the most stringent photometric constraints on Trojan companions to HD 209458b come from the continuous photometry of the system for 14 days by the MOST satellite (Rowe et al. 2006). When heavily binned into $\simeq 2 \mathrm{hr}$ intervals, this photometry has a fractional uncertainty of $\simeq 3 \times 10^{-4}$ (Rowe et al. 2006). Assuming an average density equal to that of Earth, this corresponds to a $3-\sigma$ detection threshold of $48 M_{\oplus}$. It is not clear whether the data reduction techniques used in their analysis of HD 209458 might subtract part of the signal due to a Trojan (Rowe et al. 2006). Regardless, if a Trojan had a vertical libration amplitude greater than $\simeq 9^{\circ}$, it would not always transit the star. Since the putative libration period of $\simeq 53 \mathrm{~d}$ (Murray \& Dermott 2000) is significantly longer than the duration of the MOST observations, it could have missed even a much larger Trojan.

In a sense, our method is most similar and complementary to the recently-proposed method of searching for gravitational perturbations due to low mass planets using transit timing (Holman \& Murray 2005; Agol et al. 2005). In contrast to the transit timing method, the unique geometry of Trojan orbits results in a nearly constant perturbation (assuming small amplitude libration about L4/L5) that recurs at every transit. In principle, it is not necessary to make precise measurements of the time of many transits to search for a complex pattern of perturbations. Thus, our method can be practically applied to transiting planets with long periods. Nevertheless, multiple transits should be observed to ensure that the same offset is observed and avoid potential confusion with perturbations from a more distant planet (Holman \& Murray 2005; Agol et al. 2005).

In principle, our technique could be applied to search for terrestrial-mass Trojans of giant planets orbiting in the habitable zone of their stars (Ji et al. 2005; Schwarz et al. 2005). While present search techniques are strongly biased towards finding transiting planets at short orbital periods, future space missions (e.g., Corot, Kepler) offer the prospect of finding transiting planets in the habitable zone of their stars, particularly for low mass stars where the habitable zone can be $\simeq 0.015 \mathrm{AU}$ away from the star.

We thank E. Agol, T. Beatty, E. Chiang, D. Fabrycky, M. Holman, R. Nelson, and J. Winn for helpful comments. We thank Joe Harrington for providing the time of secondary eclipse for HD 149026b. Support for E.B.F. was provided by a Miller Research Fellowship and by NASA through Hubble Fellowship grant HST-HF-01195.01A awarded by the Space Telescope Science Institute, which is operated by the Association of Universities for Research in Astronomy, Inc., for NASA, under contract NAS 5-26555. Support for B.S.G. was provided by a Menzel Fellowship from Harvard 
College Observatory.

\section{REFERENCES}

Agol, E., Steffen, J., Sari, R., \& Clarkson, W. 2005, MNRAS, 359, 567

Alonso, R., et al. 2004, ApJ, 613, L153

Bakos, G. A., et al. 2006a, preprint (astro-ph/0603291)

Bakos, G. A., et al. 2006b, preprint (astro-ph/0609369)

Bouchy, F., et al. 2005, A\&A, 444, L15

Brown, T. M., Charbonneau, D., Gilliland, R. L., Noyes, R. W., \& Burrows, A. 2001, ApJ, 552, 699

Butler, R. P., et al. 2006, ApJ, 646, 505

Charbonneau, D., et al. 2006, ApJ, 636, 445

Chiang, E. I., \& Lithwick, Y. 2005, ApJ, 628, 520

Cresswell, P., \& Nelson, R. P. 2006, A\&A, 450, 833

Deming, D., Seager, S., Richardson, L. J., \& Harrington, J. 2005, Nature, 434, 740

Ford, E. B. 2005, AJ, 129, 1706

Ford, E. B. 2006, ApJ, 642, 505

Ford, E. B., \& Chiang, E. I. 2006, in prep.

Ford, E. B., \& Rasio, F. A. 2006, ApJ, 638, L45

Gaudi, B. S. 2003, preprint (astro-ph/0307280)

Gaudi, B. S., \& Winn, J. N. 2006, preprint astro-ph/0608071)

Goździewski, K., \& Konacki, M. 2006, ApJ, 647, 573

Gregory, P. C. 2005, ApJ, 631, 1198

Holman, M. J., \& Murray, N. W. 2005, Science, 307, 1288

Holman, M. J., et al. 2006, preprint (astro-ph/0607571)

Ji, J., Liu, L., Kinoshita, H., \& Li, G. 2005, ApJ, 631, 1191 
Knutson, H., Charbonneau, D., Noyes, R. W., Brown, T. M., \& Gilliland, R. L. 2006, preprint (astro-ph/0603542)

Kortenkamp, S. J., Malhotra, R., \& Michtchenko, T. 2004, Icarus, 167, 347

Laughlin, G., \& Chambers, J. E. 2002, AJ, 124, 592

Michtchenko, T.A. \& Malhotra, R. 2004, Icarus, 168, 237.

McCullough, P.R. et al. 2006, ApJ, 648, 1228.

Morbidelli, A., Levison, H. F., Tsiganis, K., \& Gomes, R. 2005, Nature, 435, 462

Murray, C. D., \& Dermott, S. F. 2000, Solar System Dynamics, Cambridge, UK: Cambridge University Press.

Nesvorný, D., \& Dones, L. 2002, Icarus, 160, 271

Nesvorný, D., Thomas, F., Ferraz-Mello, S., \& Morbidelli, A. 2002, Celestial Mechanics and Dynamical Astronomy, 82, 323

O’Donovan, F.R. et al. 2006, ApJL, in press

Rasio, F. A., Tout, C. A., Lubow, S. H., \& Livio, M. 1996, ApJ, 470, 1187

Rasio, F. A., \& Ford, E. B. 1996, Science, 274, 954

Rowe, J. F., et al. 2006, ApJ, 646, 1241

Sato, B., et al. 2005, ApJ, 633, 465

Schwarz, R., Pilat-Lohinger, E., Dvorak, R., Érdi, B., \& Sándor, Z. 2005, Astrobiology, 5, 579

Thommes, E. W. 2005, ApJ, 626, 1033

Winn, J. N., et al. 2005, ApJ, 631, 1215

Wright, J. T. 2005, PASP, 117, 657

Wu, Y., \& Murray, N. 2003, ApJ, 589, 605 
Table 1. Sensitivity to Trojans of Extrasolar Planets

\begin{tabular}{lcccccccc}
\hline \hline \multicolumn{1}{c}{ Star } & $\mathrm{P}$ & $\mathrm{K}$ & $\begin{array}{c}\sigma_{R V^{\mathrm{a}}} \\
(\mathrm{d})\end{array}$ & $N_{R V}$ & $\begin{array}{c}M_{\star} \\
\left.\left(\mathrm{m} \mathrm{s}_{\odot}\right)^{-1}\right)\end{array}$ & $\begin{array}{c}\sigma_{\Delta t} \mathrm{~b} \\
(\mathrm{~min})\end{array}$ & $\begin{array}{c}\sigma_{m_{T}}^{\mathrm{b}} \\
\left(M_{\oplus}\right)\end{array}$ & References \\
\hline HD 209458 & $3.5247455(2)$ & $84(1)$ & 5.0 & 64 & 1.101 & 8.5 & 2.6 & 1,2 \\
HD 149026 & $2.87598(1)$ & $43(2)$ & 5.7 & 16 & 1.30 & 30.9 & 6.2 & $1,3,4,5$ \\
HAT-P-1 & $4.46529(9)$ & $60(2)$ & 5.1 & 13 & 1.11 & 34.0 & 6.4 & 6 \\
TrES-2 & $2.47063(1)$ & $181(3)$ & 6.9 & 11 & 1.08 & 9.2 & 7.6 & 7 \\
HD 189733 & $2.21857(2)$ & $205(6)$ & 15 & 24 & 0.82 & 10.7 & 8.9 & 8,9 \\
TrES-1 & $3.030065(8)$ & $115(6)$ & 14 & 8 & 0.89 & 42.3 & 17. & 1,10 \\
XO-1 & $3.94153(3)$ & $116(9)$ & 15 & 6 & 1.0 & 67.4 & 25. & 11,12 \\
\hline
\end{tabular}

${ }^{a}$ When available, we list the rms velocity to the published best-fit RV model rather than the quoted measurement uncertainty.

${ }^{b}$ We list " $1-\sigma$ " uncertainties, implicitly assuming circular orbits for the transiting planets.

References. - (1) Butler et al. 2006; (2) Knutson et al. 2006; (3) Charbonneau et al. 2006; (4) Sato et al. 2005; (5) Harrington et al. 2006; (6) Bakos et al. 2006b; (7) O'Donovan et al. 2006; (8) Bouchy et al. 2005; (9) Bakos et al. 2006a; (10) Alonso et al. 2004; (11) McCullough et al. 2006; (12) Holman et al. 2006 
A

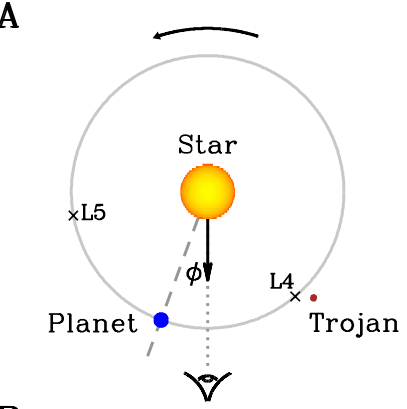

B

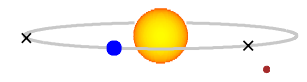

C

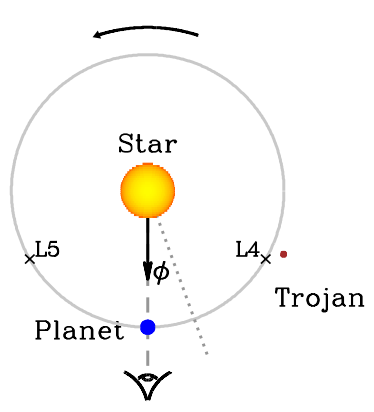

D

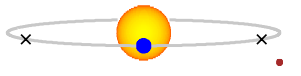

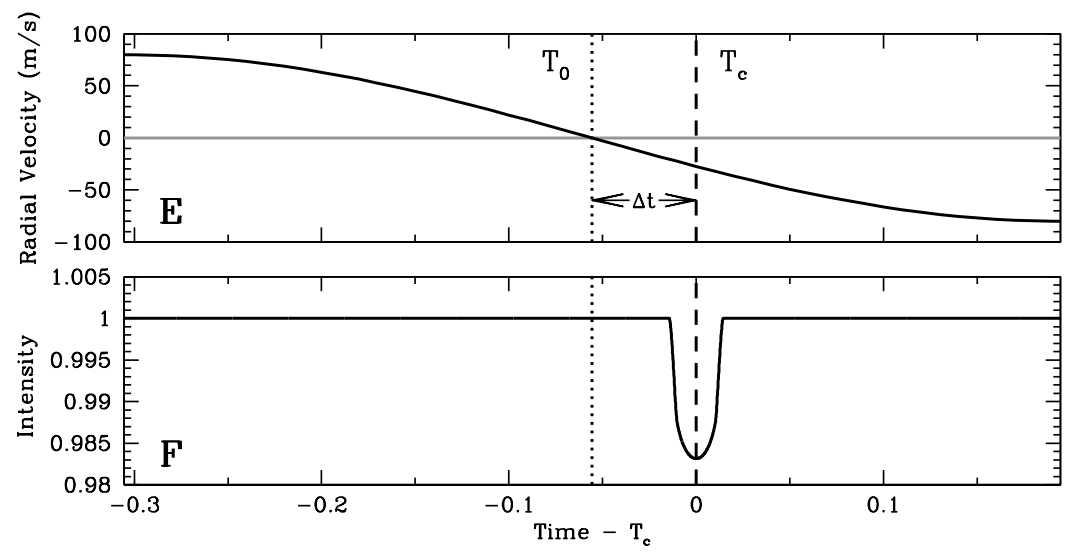

Fig. 1. - Illustration of the method to detect Trojan companions of transiting planets by comparing the transit and RV observations. Views of the star, planet and Trojan (A,C plan, B,D from observer's perspective; not to scale). The grey circle shows the orbit of the planet and Trojan. The dotted line indicates the direction of the acceleration of the star, the dashed line the direction of the transiting planet, and $\phi$ is the angle between these two directions. The vector shows the direction toward the observer. Panels $(\mathbf{A}, \mathbf{B})$ show the position at $T_{0}$, the time of the stellar reflex RV null. Panels $(\mathbf{C , D})$ show the position at $T_{c}$, the time of the central transit. Panel $(\mathbf{E})$ shows the stellar reflex RV as a function of time (in units of the period of the planet), with the times $T_{0}$ and $T_{c}$ indicated. Panel $(\mathbf{F})$ shows the intensity of the star as a function of time. We have assumed that the Trojan is inclined so that it does not transit the parent star. 


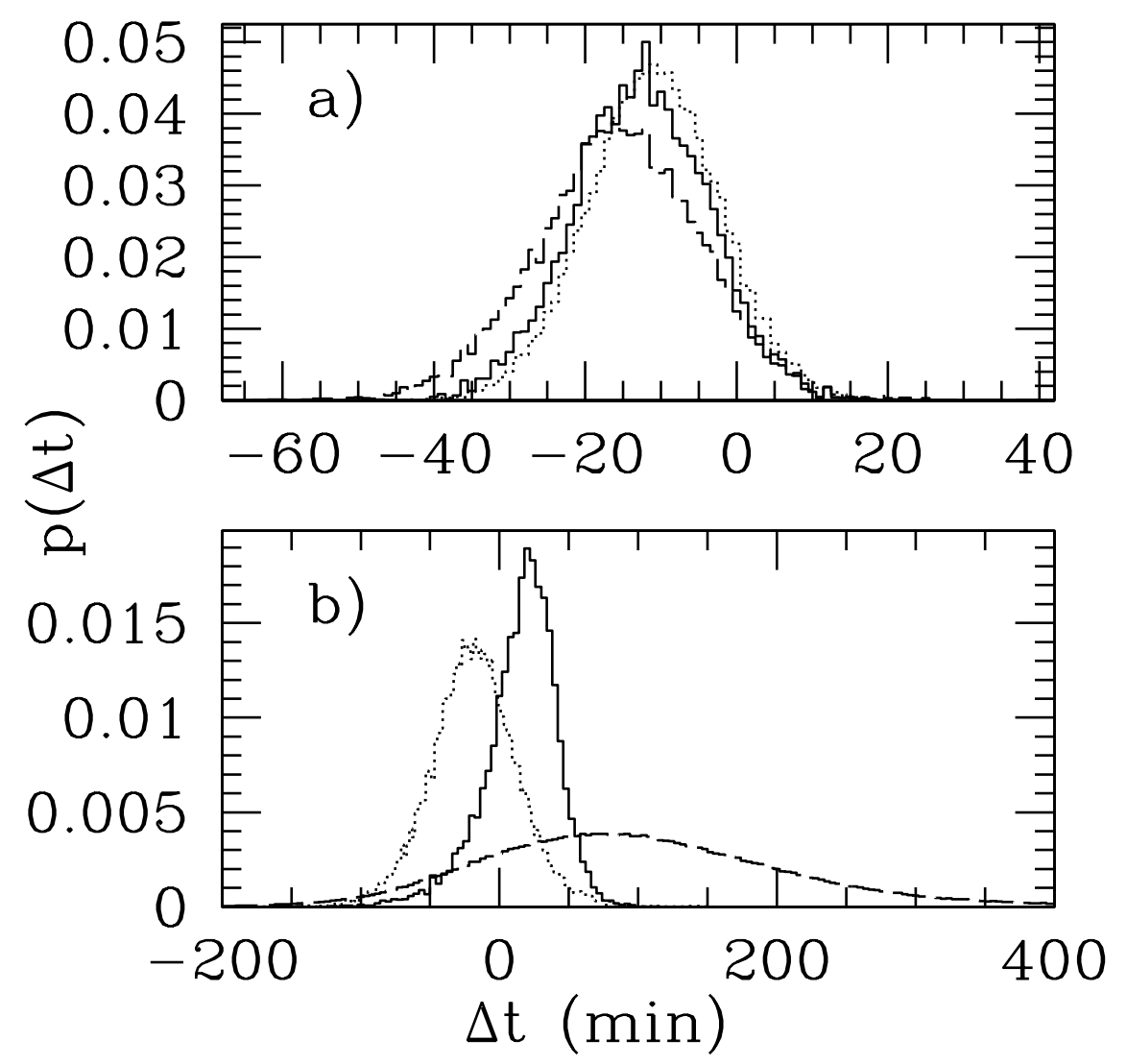

Fig. 2.- Marginal posterior probability distributions of $\Delta t$ for HD 209458b (top) and HD 149026b (bottom). Here $\Delta t \simeq\left(M_{0}-e \cos \omega\right) P /(2 \pi)$ is the difference between the time of the stellar reflex RV null and the time of central transit that could be due to a Trojan. The dotted curves assume a circular orbit, the dashed curves allow for a non-circular orbit (ignoring the constraint from the secondary eclipse), and the solid curves allow for a non-circular orbit and incorporates the measured times of secondary eclipse. 